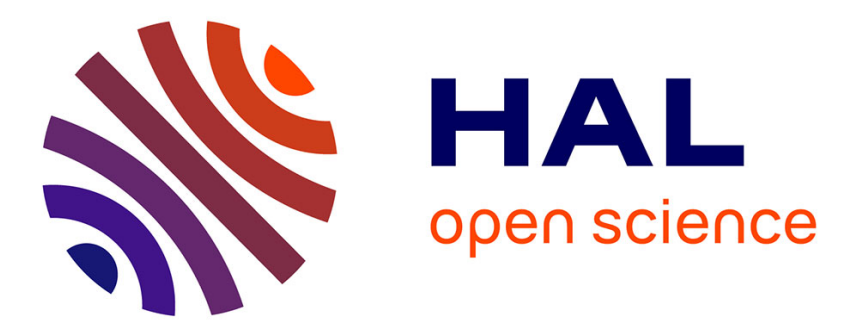

\title{
Optimization of the performances of SU-8 organic microcantilever resonators by tuning the viscoelastic properties of the polymer
}

Georges Dubourg, Isabelle Dufour, Claude Pellet, Cédric Ayela

\section{To cite this version:}

Georges Dubourg, Isabelle Dufour, Claude Pellet, Cédric Ayela. Optimization of the performances of SU-8 organic microcantilever resonators by tuning the viscoelastic properties of the polymer. Sensors and Actuators B: Chemical, 2012, 169, pp.320-326. 10.1016/j.snb.2012.04.088 . hal-00694343

\section{HAL Id: hal-00694343 \\ https://hal.science/hal-00694343}

Submitted on 13 Nov 2014

HAL is a multi-disciplinary open access archive for the deposit and dissemination of scientific research documents, whether they are published or not. The documents may come from teaching and research institutions in France or abroad, or from public or private research centers.
L'archive ouverte pluridisciplinaire HAL, est destinée au dépôt et à la diffusion de documents scientifiques de niveau recherche, publiés ou non, émanant des établissements d'enseignement et de recherche français ou étrangers, des laboratoires publics ou privés. 
Optimization of the performances of $\mathrm{SU}-8$ organic microcantilever resonators by tuning the viscoelastic properties of the polymer.

\title{
G. Dubourg, I. Dufour, C. Pellet and C. Ayela
}

Univ. de Bordeaux, CNRS UMR 5218, IMS, 351 Cours de la Libération F-33400 Talence Cedex, France.

\author{
Corresponding author: \\ Georges Dubourg \\ georges.dubourg@ims-bordeaux.fr \\ Tel: +33 (0)5 40008250
}

\begin{abstract}
This paper describes a promising method to improve the performances of organic resonant microcantilevers. This method is based on the tuning of the viscoelastic properties of the material by controlling internally the temperature of the structure. Actuation of the SU-8 free-standing microstructures has been integrated by means of electromagnetic forces while a static resistive thermal effect, obtained by applying a DC current in a conducting path, has been used to control the temperature of the structure. Investigation of the electromagnetic and thermal influence on the static and dynamic behavior has shown that the thermal effect dominates the electromagnetic one in static mode and inversely in dynamic mode. As a result, by applying a constant heating of the structure, the viscoelastic properties of polymers have been tuned, and the quality factor of SU-8 microcantilevers has been thereby enhanced by a factor 2 in air and 2.25 in liquid.
\end{abstract}

Keywords: organic microresonator; SU-8; viscoelastic properties; Q-enhancement.

\section{Introduction}

Free-standing microcantilevers are widely used as micro- and-nanoelectromechanical systems (MEMS and NEMS). More specifically, silicon cantilevers have shown great potential for sensing applications such as, molecular recognition [1,2] or virus detection [3]. However, a recent emerging alternative concerns the introduction of polymers as structural layer in MEMS resonators [4], actuators [5] and spring structures [6] due to the ease of their processing, their low cost, their low temperature requirement during fabrication, and the possible tuning of mechanical properties such as Young modulus, a quality criterion hard to be achieved when using silicon.

When organic MEMS are used in static mode, one of the advantages is their softness resulting in large deflections for small external applied forces. This specific property has been used for the fabrication of sensors used for chemical or biological applications [7, 8,9] and micromanipulators for cell handling $[10,11]$. In dynamic mode, it is well-known that the quality factor $\mathrm{Q}$ is an important parameter for the resonance characteristics. The quality factor determines the sharpness of the resonant peak and thus the frequency stability of the resonator. Therefore, a good quality factor is crucial for most resonator applications. In this context, the low quality factor limits the actual performance of organic MEMS resonators since losses in organic materials induce a non-negligible material damping. This raises the question of how the quality factor of polymer resonant microcantilevers can be improved. Recently, various methods have been proposed. For instance, in order to counteract the negative effect of the relative low quality factor, an electronic positive feedback has been used for polymer microcantilevers corresponding to an artificial increase of Q [4]. Other promising methods consist in using microcantilevers in the in-plane modes including longitudinal and lateral ones $[12,13]$ or introducing a microchannel into the cantilever to reduce the fluid damping and thus, to improve the quality factor for applications in liquid media [14]. Recently, the fabrication of asymmetric anchors or electrodes has been proposed to enhance the sensitivity of microcantilevers operating in the flexural modes $[15,16]$. However, the fabrication of such devices generally requires more complex fabrication steps, reducing the simplicity and low cost criteria associated to organic MEMS. The control of the mechanical properties of silicon cantilevers by self-oscillations can be also realized [17] but this method is not adapted to organic structures due to their different mechanical behavior. 
In this context, the understanding of intrinsic dissipation effects occurring in polymer materials and its influence on the damping behavior of polymer microresonators is of great interest since this behavior affects directly the Q-factor. Investigations on origins of damping in SU-8 microcantilevers have demonstrated that material damping due to polymer viscoelastic properties is the main loss mechanism [18]. The influence of the temperature on the viscoelastic properties of SU-8 has been also reported [18].

In the present work, a simple method to precisely control the viscoelastic properties of SU-8 by inducing an internal heating is proposed in order to improve the quality factor of SU-8 resonators. This is achieved by patterning a U-shaped conducting path on top of the cantilever surface, allowing a constant static resistive heating thanks to a DC current flowing through the conducting path. Also, an integrated dynamic actuation of the cantilevers is provided by electromagnetic forces due to its simple implementation, the only criterion being an alternative current flowing into the conducting path and an external magnetic field. With this approach, the performances of resonant SU-8 microcantilevers have been evaluated and the dependence of the viscoelastic properties of SU-8 on Q-factor is reported. For a better control of the viscoelastic properties, the influence of the electrothermal, electrothermomechanical and electromagnetic effects on the static and the dynamic behavior of the structures has been widely investigated. As a result, the viscoelastic properties of the polymer have been successfully controlled thermally and the quality factor of SU-8 resonators has been significantly improved in air and in liquid media, where values of $\mathrm{Q}$ similar to those of silicon-based microcantilevers have been obtained.

\section{Materials and methods}

\subsection{Fabrication process}

The specific polymer used in this study is SU-8, an epoxy-based negative photoresist (SU-8® 3000) from MicroChem, that ensures different functions: structural, support and adhesive layer. SU-8 was chosen for its excellent processability, and its properties which have been intensively studied [19].

The fabrication process of the microcantilever is based on a method inspired from wafer bonding. It consists in structuring simultaneously two wafers where the cantilever structures are first patterned upside down on a silicon carrier wafer and then clamped on a receiver wafer as reported in our previous work [20].

First, a silicon carrier substrate has been coated with an Omnicoat ${ }^{\circledR}$ sacrificial layer (from MicroChem) to allow the release of the microstructures after the transferring step. Then, SU-8 microstructures and supporting layers have been successively spin-coated and patterned by photolithography. In parallel, the SU-8 transferring layer is structured on another silicon substrate. Next, both substrates are bonded together by a thermal wafer-bonding method. Afterwards, the freestanding organic structures are released by chemical etching of the Omnicoat ${ }^{\circledR}$ sacrificial layer.

To ensure the integrated actuation of the organic structures, a gold path has been deposited between the sacrificial layer and the SU-8 structural one. Standard methods for gold patterning such as lift-off or etching are not appropriate in our case since the Omnicoat ${ }^{\circledR}$ sacrificial layer does not resist to the solvents used for the protective resists patterning generally used in such processes. Hence, an alternative method based on shadow-masking has been chosen since it does not require any chemical etching step. This method combines deposition and patterning in one step via thermal evaporation of gold through polymeric microstencils made of SU-8 following the method described in [20].

The resulting gold conducting path, characterized by a thickness of $100 \mathrm{~nm}$, and integrated on the organic structures (cf. Fig. 1) allows the integrated actuation and the thermal control of the SU-8 cantilevers as described in the next section. To finish the process, the free-standing structures are mounted on a PCB. The conducting lines of the PCB are wire-bonded to pads present on the structure. With this process, matrices of organic cantilever resonators have been successfully realized, as shown on Fig. 1.

\subsection{Experiments}

Polymer microstructures can be actuated by several methods including electrostatic [4], 
electromagnetic [21] or electrothermal actuation [22]. In this paper, we propose an electromagnetic dynamical actuation coupled with an integrated resistive heating by electrothermal effects.

The dynamic actuation of the polymer-based microcantilevers is generated by an electromagnetic Laplace force. For this, the structures are mounted above an external magnet, and the Laplace force is then exerted by an alternative electrical current (AC) flowing through the gold path deposited on the structure. Fig. 2 shows a schematic of the cantilever actuated by the Laplace force (Fig. 2). This configuration offers the possibility to obtain the torsional and flexural resonant modes. The dynamic characteristics of the organic microcantilevers have been then recorded with a laser-Doppler vibrometer MSA-500 from Polytec measuring nanometer magnitude displacements.

A continue electrical current (DC) can be also applied on the conducting path which can generate a static Laplace force and a heating of the resistor by Joule effect. In our case, the temperature rise associated with the resistive heating has been used for a precise control of the temperature in the cantilever. For instance, an applied DC power of $3.5 \mathrm{~mW}$ gives an internal resistive heating of the structure of $90^{\circ} \mathrm{C}$ (this temperature results from Comsol simulation and thermal resistor measurements). This temperature rise induces also a thermomechanical deflection of the structure due to the bimorph effect from the Au conducting path coated on top of structured SU-8. This effect can be added to the microcantilever deflection resulting from the Laplace Force. The microcantilever deflection due to both static effects has been measured thanks to an interferometry optical profiler NT9080 from Veeco.

By using both approaches simultaneously, the organic microstructures can be used at different resonant frequencies while applying an initial resistive heating of the organic material.

\section{Results and discussion}

\subsection{Dynamic characterization of the microstructures}

To obtain a reliable organic microresonator, it is important to evaluate the actual performances of the microstructures. An accurate dynamic characterization of the organic microcantilevers including mechanical resonant frequencies and quality factors is thus required.

\subsubsection{Resonant frequencies}

Cantilever resonant frequencies have been obtained by applying an alternative current $\left(\mathrm{I}_{\max }=40 \mu \mathrm{A}\right)$ to the gold conducting path, that is low enough to prevent from a dynamic electrothermal effect. The mechanical properties of the material defined by the Young's modulus of the structured layer have been then deduced from the out-of-plane flexural resonant modes using Eq. (1) [23].

(1) $E=\left(4 \pi^{2} f_{n}^{2} L^{4} 12 \rho\right) /\left(\lambda^{4} h^{2}\right)$

In this Eq., $E$ represents the cantilever effective Young's modulus, $f_{n}$ is the resonance frequency of the $\mathrm{n}^{\text {th }}$ mode, $\mathrm{L}$ and $\mathrm{h}$ are respectively the length and thickness of the microcantilever, $\rho$ represents the mass density of the material, and $\lambda_{n}$ the eigenvalue of the $\mathrm{n}^{\text {th }}$ resonance mode.

Fig. 3 shows the Young's modulus evolution measured for different flexural resonant modes. First, a wide panel of out-of-plane resonant modes, including torsional and flexural ones can be observed due to the high flexibility of the organic structures. In some cases (data not shown), the first fifteen flexural modes and torsional modes have been observed with the vibrometer. For the flexural modes, the low values of Young's modulus obtained are in agreement with the literature [24], validating the method used for its calculation. A slight increase of E can be also observed with the frequency. In fact, it is known that SU-8 provides a viscoelastic behavior [25], meaning that the mechanical properties of the material are frequency dependent and can be expressed by a complex modulus: 
(2) $E^{*}=E^{\prime}+\mathrm{i} E^{\prime,}$

Where $E^{\prime}$, also called storage modulus, is the real part of $\mathrm{E}^{*}$, in phase with the strain. $E^{\prime}$, the loss modulus, is the imaginary part of $E^{*}$, out-of-phase with the strain. E' represents the stiffness of the material, and E" the loss due to material damping. It is thus noteworthy to consider the material viscoelastic properties which strongly influence the quality factor of the organic microresonators, as shown in the next paragraphs.

\subsubsection{Quality factors}

The ability of resonant structures to be used as highly mass sensitive devices depends mainly on their quality factor which defines losses in the structure. Thereby, the quality factor of SU-8 microresonators has been determined by using the $-3 \mathrm{~dB}$ bandwidth of the resonant frequency spectra. From Fig. 4, it can be seen that the organic cantilevers have relatively low quality factors in air, decreasing with the frequency. For instance, the quality factor of a structure characterized by a length of $380 \mu \mathrm{m}$ decreases from 30 at low frequencies to around 10 at high frequencies (above $1 \mathrm{MHz}$ ). Quality factor depends on several loss sources including losses from the clamped edge, the surface losses, the air damping and internal material damping. But, in the case of organic microresonators, the actuation of the structures induces vibrations of a viscoelastic material. Losses due to viscoelastic material damping can be expressed by Eq. (3) [18]:

(3) $1 / Q_{\text {viscoelast. }}=E^{\prime \prime} / E^{\prime}=\tan \delta$

Where $\tan \delta$, the loss tangent, represents the phase angle between stress and strain.

Furthermore, previous works [18] have proved that the dominant damping mechanism is the viscoelastic material damping for SU-8 microresonators. Thereby, the quality factor of the organic microstructures can be expressed by Eq. (4):

(4) $Q=E^{\prime} / E^{\prime \prime}=1 / \tan \delta$

As shown by Eq. 4, the quality factor of SU-8 microresonators is inversely proportional to the loss tangent. The tuning of viscoelastic properties of the material could offer the possibility to enhance the quality factors of the organic microcantilevers and thus, their performances as microresonators.

\subsection{Optimization of the performances of SU-8 microresonators}

\subsubsection{Method}

Previous investigations on the viscoelastic behavior of SU-8 have shown the influence of the temperature on the viscoelastic properties and that the loss tangent decreases with the temperature to a minimum peak [18]. Above this temperature (around $90^{\circ} \mathrm{C}$ ), the loss tangent increases representing the glass transition. As the quality factor is inversely proportional to the loss tangent (see Eq. 4), a maximum quality factor could be observed at the temperature corresponding to the minimum peak of the loss tangent. The thermal tuning of the viscoelastic properties of the SU-8 material by controlling internally the temperature of the structure would be an efficient method to improve the quality factors of the organic SU-8 microresonators. For this to be achieved, the conducting path integrated on the top surface of the microcantilevers previously described, allows an internal resistive heating of the organic microstructure. Hence, the organic microcantilevers can be used at the resonance frequency while a constant heating can be applied by adding an offset to the sinusoidal signal. Therefore, by using an electrothermal static effect, the viscoelastic properties of the SU-8 material can be accurately tuned to enhance the performances of the organic microresonators. However, this method requires a precise knowledge on the influence of the electromagnetic and the electrothermal effect on the static and the dynamic behavior of the microcantilevers. 


\subsubsection{Static behavior of SU-8 microcantilevers}

To precisely control the temperature of the SU- 8 microcantilevers, the electrothermal effect is chosen for its simplicity and efficiency. But, due to different thermal expansion coefficients between SU-8 $(52 \mathrm{ppm} / \mathrm{K})$ and gold $(14 \mathrm{ppm} / \mathrm{K})$, a static electrothermomechanical actuation of the structures should occur. Thus, the deflection of the cantilevers due to resistive heating has been investigated by applying a constant voltage across the resistor, corresponding to the U-shaped gold conducting path. The maximum DC voltage that can be applied over the resistor of $263 \Omega$ before causing damage of the microstructure and avoiding hysteresis effects is around $2 \mathrm{~V}$ corresponding to a structure heating above the glass transition temperature of the polymer. Also, to clearly discriminate the influence of the electromagnetic and the electrothermomechanical static actuation, measurements with and without magnet have been performed. In this context, to evaluate the influence of the electromagnetic actuation on the global deflection of the microcantilevers, measurements have been performed in order to have Laplace force up and then down by applying respectively positive and negative voltages. The resulting deflections caused by the applied power in these different conditions are presented in Fig. 5, in the case of a microcantilever characterized by a length of $380 \mu \mathrm{m}$.

The measured deflections of the structure, presented on Fig. 5, are similar when only the electrothermomechanical actuation is provided and when a Laplace force is generated, either up or down. The discrepancy between the different measurements is mainly due to uncertainty caused by the high transparency of SU-8, making optical measurements hard to be achieved. Thus, from this figure, it is clear that the static electrothermomechanical actuation dominates the electromagnetic static actuation. The theoretical electrothermomechanical results performed with Finite Element Modeling (Comsol) confirm the trend of the deflection obtained experimentally. Moreover, below $1.5 \mathrm{~mW}$, the deflection of the structure is nearly zero, meaning that the thermal effect can be neglected below this power value. This result is in agreement with previous observations [22].

\subsubsection{Consequence on dynamic behavior}

In dynamic mode, the integrated actuation can result from the electrothermomechanical or/and the electromagnetic effect. In the case of electromagnetic actuation, an $\mathrm{AC}$ current having an angular frequency $\omega$ results in Laplace deflection oscillating at $\omega$. On the contrary, in the case of electrothermomechanical actuation, an AC current (without DC offset) characterized by an angular frequency of $\omega$ generates temperature oscillations in the cantilever at $2 \omega$ leading to thermomechanical deflection also at $2 \omega$ [26]. Thus, as the Laplace actuation has the same frequency as the current oscillation, this leads to a thermomechanical response equal to twice the actuation frequency. To evaluate the influence of both actuation schemes in dynamic mode, measurements have been performed with and without magnet.

From Fig. 6, it can be seen that the magnitude of the resonant peak observed with the magnet is ten times higher than the one measured without magnet. As the thermomechanical frequency is equal to twice the actual resonant frequency $\mathrm{f}$ [26], this means that the thermomechanical response should be at $2 \mathrm{f}$. Thus, the peak observed without magnet cannot result from the thermomechanical actuation but from an electromagnetic actuation caused by Earth's magnetic field. Moreover, no response has been observed at $2 \mathrm{f}$ (with and without magnet), showing that the amplitude of the AC current applied $\left(I_{\max }=30 \mu \mathrm{A}\right)$ is too low to generate enough power allowing a measurable thermomechanical deflection. Under this condition, considering the applied power, the electrothermal effects can be neglected in dynamic and the electromagnetic actuation becomes particularly appropriate for the dynamic mode. Moreover, as the oscillating deflection of highly flexible organic structures can be achieved at very low current density without heating the structure, the temperature of the structure can be precisely tuned by the static electrothermal effect only.

Otherwise, an increase of the temperature due to an internal heating affects the mechanical properties of the SU-8 material and thus the resonant frequencies of the structures (see Eq. (1)). Therefore, this effect has to be considered in the dynamic behavior. From Fig. 7, a decrease of the resonant frequency as a function of the applied DC power and thus the temperature can be observed. One could think that initiating a static deflection into the cantilevers would increase their stiffness and thus their resonant frequencies. But in present case, the specific decrease of the resonant frequency is due to the softening 
of the SU-8 material caused by a decrease of E' with the temperature [25]. Moreover, the shape of the curves observed in Fig. 7 is close to the thermal behavior of the elastic part of the complex Young's modulus [25], confirming the thermal dependence of the SU-8 cantilevers with the one of E' of SU-8. Indeed, an important drop of the frequency can be observed between 3 and $5 \mathrm{~mW}$ which should correspond to the beginning of the glass transition of SU-8.

\subsubsection{Q-factor optimization in air}

It has been seen previously that the viscoelastic properties of SU-8 depend on the temperature. By analyzing previous works from the literature [18], the quality factor could show a maximum peak at a temperature below the one of the material glass transition. To study the influence of the structure heating on the quality factor, an offset voltage has been applied into the conducting path. One has to remind that although the static electrothermomechanical actuation of the cantilevers has been studied in this work, the main idea was to induce precisely an internal heating into the SU-8 material.

Fig. 8 shows the quality factor measurements for different DC powers applied to a microcantilever characterized by a length of $380 \mu \mathrm{m}$. For each step, measurement stability is reached ensuring a constant temperature in the structure. The evolution of the quality factor is as predicted and in agreement with the thermal evolution of the loss tangent reported in [18]. Indeed, the quality factor increases until a maximum peak found at $90^{\circ} \mathrm{C}$ and decreases above this temperature which corresponds to the beginning of the glass transition of the material. In this case, the glass temperature is not exceeded. Irreversible modifications of the materials properties due to an internal heating are thus avoided ensuring repeatability of the measurements. An increase up to $100 \%$ of the quality factor is observed for the second resonant flexural mode. But, it has been observed that the resonant frequency decreases with the applied DC power (see Fig. 7); therefore, it can be assumed that the Q rise is not due to the increase of the stiffness of the organic structure (as often reported to improve the quality factor of resonators [27]). This rise is caused by a larger thermal decrease of the loss modulus, E", in comparison to the decrease of the storage modulus, E', resulting in an increase of the E'/E" ratio and therefore Q, until $3.7 \mathrm{~mW}$. Also, from Fig. 8 it can be seen that the quality factor of the second mode increases more than the first mode since an increase of $25 \%$ and $100 \%$ has been observed for the first and the second mode respectively.

\subsubsection{Q-factor optimization in liquid}

In liquid, losses due to the fluid damping dominate over internal material losses for silicon based microstructures. In air, Q-factors up to 1500 have been measured for the first out-of-plane bending mode [28]. In water, Q-factors of silicon-based microcantilevers rarely exceed 2-20 due to the substantial viscous damping caused by the fluid. However in the case of an organic microresonator, the effects of viscoelastic material damping have to be considered too. The tuning of the viscoelastic properties of the organic structures could have the potential to enhance the quality factor of the cantilevers in liquid. It is thus interesting to investigate the effects of the initial heating of the structure on its quality factor in liquid media. The liquid used in this study is dodecane, a non-conducting fluid characterized by a viscosity of $1.55 \mathrm{cP}$ and a density of $746 \mathrm{~kg} / \mathrm{m}^{3}$ at $25^{\circ} \mathrm{C}$. Fig. 9 shows the evolution of the quality factor of an SU-8 organic microstructure characterized by a length of $380 \mu \mathrm{m}$ for increasing DC power values.

A maximum peak of $120 \%$ can be observed for the second flexural resonant mode, for an applied power of $3.5 \mathrm{~mW}$ corresponding to a temperature of $90^{\circ} \mathrm{C}$, as already observed in air. Although in liquid the losses due to the environment cannot be neglected, the influence of the material damping remains important. In our case, the quality factor increases from 4.8 to 10.8 , the latter one being similar to the values obtained for silicon cantilevers. But a greater rise can be observed in liquid $(120 \%)$ than in air (100\%). In liquid, losses due to fluid damping have to be considered. These losses decrease with the density and the viscosity of the fluid [29, 30]. Moreover, the viscosity and the density of the dodecane decrease with the temperature [31]. Therefore, the structure heating reduces the fluid damping and thus increases also the quality factor. The combined decrease of both material and fluid damping leads to a more substantial rise of the quality-factor in liquid than in air where only the material damping decrease is considered. However, by simply integrating a conducting path onto 
SU-8 organic microcantilevers, used as internal heater, a significant improvement of their quality factor is successfully achieved. These results potentiate organic MEMS resonators as good candidates for future biochemical mass sensitive applications in liquid media.

\section{Conclusion}

A promising method for the improvement of the performances of organic microcantilever resonators has been demonstrated. This method consists in precisely controlling the viscoelastic properties responsible of the low quality factor of the polymer devices by applying a constant heating of the structures. For this, an electrothermal effect is generated simply by incorporating a conducting path on top of the SU-8 structural layer. But the conducting path has been used also for an electromagnetic dynamic actuation by mounting the cantilever chips above a magnet.

The dynamic characterization of these organic structures has been achieved to evaluate the performances of organic resonators including mechanical resonant frequencies and quality factors. Low Young's modulus values of highly flexible organic structures have been obtained thanks to the flexural resonant frequencies. Investigations on the electrothermal and electromagnetic effect on the static and dynamic behavior of the cantilevers have been realized. This study has shown that the static resistive heating is an efficient method to control the temperature of the structure while the electromagnetic actuation is an appropriate method to create integrated structure oscillations. With this approach, the performances of the organic microcantilever resonators have been successfully improved. The quality factor of the resonant microcantilevers has been enhanced by $100 \%$ in air and $120 \%$ in liquid. These structures have thus the potential to be used for biosensing applications in liquid media. Future works will aim to find polymer materials having tunable viscoelastic properties allowing this method to be used at lower temperatures.

\section{References}

[1] T.P. Burg, A.R. Mirza, N. Milovic, C.H. Tsau, G.A. Popescu, J.S. Foster, S.R. Manalis, VacuumPackaged Suspended Microchannel Resonant Mass Sensor for Biomolecular Detection, J. Microelectromech. Syst. 15 (2002) 1466-1476.

[2] T.P. Burg, M. Godin, S.M. Knudsen, W. Shen, G Carlson, J.S. Foster, K. Babcock, S.R. Manalis, Weighing of biomolecules, single cells and single nanoparticles in fluid, Nature 446 (2007) 10661069.

[3] K.S. Hwang, S.M. Lee, K. Eom, J.H. Lee, Y.S. Lee, J.H. Park, D.S. Yoon, T.S.Kim, Nanomechanical microcantilever operated in vibration modes with use of RNA aptamer as receptor molecules for label-free detection of HCV helicase, Biosensors and Bioelectronics 23 (2007) 459-465.

[4] S. Schmid, P. Senn, C. Hierold, Electrostatically actuated nonconductive polymer microresonators in gaseous and aqueous environment, Sensors Actuators A 145-146 (2008) 443-448.

[5] Y. Kato, T. Sekitani, M.T. takamiya, M. Doi, K. Asaka, T. Sakurai, T. Someya, Sheet-type Braille displays by integrating organic field-effect transistors and polymeric actuators, IEEE Trans. Electron Devices 54 (2007) 202-209.

[6] D. Bachmann, B. Schoberle, S. Kuhne, Y. Leiner, C. Hierold, Fabrication and characterization of folded SU-8 suspensions for MEMS applications, Sensors and Actuators A 130-131 (2006) 379-386.

[7] J. Fritz, M. K. Baller, H. P. Lang, H. Rothuizen, P. Vettiger, E. Meyer, H.J. Güntherodt, C. Gerber, J. K. Gimzewski, Translating Biomolecular Recognition into Nanomechanics, Nature 288 (2000) 316-318.

[8] M. Calleja, P. Rasmussen, A. Johansson, A. Boisen, Polymeric Mechanical Sensors with Integrated Readout in a Microfluidic System, Proc. SPIE 5116, 314, (2003) 314-321. 
[9] M. Calleja, J. Tamayo, A. Johansson, P. Rasmussen, L. Lechuga, A. Boisen, Polymeric Cantilever Arrays for Biosensing Applications, Sens. Lett. 1 (2003) 20.

[10] E. W. H. Jager, E. Smela, O. Inganas, Microfabricating Conjugated Polymer Actuators, Science $290(2000) 1540$.

[11] N. Chronis, L. P. Lee, Electrothermally Activated SU-8 Microgripper for Single Cell Manipulation in Solution, J. Microelectromech. Syst. 14 (2005) 857.

[12] C. Castille, I. Dufour, C. Lucat, Longitudinal vibration mode of piezoelectric thick-film cantilever-based sensors in liquid media, Appl. Phys. Lett. 96 (2010) 154102.

[13] R. Cox, F. Josse, S. M. Heinrich, O.Brand, I. Dufour, Characteristics of laterally vibrating resonant microcantilevers in viscous liquid media, J. Appl. Phys. 111(2012) 014907.

[14] T. P. Burg, S. R. Manalis, Suspended microchannel resonators for biomolecular detection,Appl. Phys. Lett. 83 (2003) 2698-2700.

[15] H. Sharma, D. Nguyen, A. Chen, V. Lew, M. Khine, Unconventional Low-Cost Fabrication and Patterning Techniques for Point of Care Diagnostics, Sensors Actuators B, 153 (2011) 64-70.

[16] B.N Johnson, R.Mutharasan, Expression of picogram sensitive bending modes in piezoelectric cantilever sensors with nonuniform electric fields generated by asymmetric electrodes Rev. Sci. Instrum. 81 (2010) 125108.

[17] C.C Hohberger, K.Karrai, Self-oscillation of micromechanical resonators 4th IEEE Inter. Conf. on Nanotech. (2004) 419-421.

[18] S. Schmid, C. Hierold, Damping mechanisms of single-clamped and prestressed double clamped resonant polymer microbeams, J. Appl. Phys. 104 (2008) 093516.

[19] H. Lorenz, M. Despont, N. Fahrni, J. Brugger, P. Vettiger, P. Renaud, High-aspect-ratio, ultrathick, negative-tone near-UV photoresist and its applications for MEMS, Sens. Actuators A 64 (1998) 33.

[20] G. Dubourg, L.Fadel-Taris, I. Dufour, C. Pellet, C.Ayela, Collective fabrication of all-organic microcantilever chips based on a hierarchical combination of shadow-masking and wafer-bonding processing methods, J. Micromech. Microeng. 21 (2011) 095021.

[21 M. Im, I.J. Cho, K.S. Yun, E. Yoon, Electromagnetic actuation and microchannel engineering of a polymer micropen array integrated with microchannels and sample reservoirs for biological assay patterning, Applied physics letters 91 (2007) 124101

[22] X. Zhang, Y. Wu, X. Miao, C. Zhang, G. Ding, An Electro thermal SU-8 Cantilever, Micro Actuator based on Bimorph Effect, Nano/Micro Engineered and Molecular Systems (NEMS), 5th IEEE International Conference (2010) $362-365$.

[23] R.D. Blevins, Formulas for natural frequency and mode shape, Van Nostrand Reinhold Company, 1979.

[24] N. Ferrell, D. Hansford, Microfabrication and nanomechanical characterization of polymer microelectromechanical system for biological applications, Journal of vacuum science and technology, 23(4) (2005) 811-819. 
[25] K. Wouters, P. Gijsenbergh, R. Puers, Comparison of methods for the mechanical characterization of polymers for MEMS applications, J. Micromech. Microeng. 21 (2011) 115027

[26] B. Lee, C. B. Prater, W.P. King, Lorentz force actuation of a heated atomic force microscope cantilever, Nanotechnology 23 (2012) 055709.

[27] Q. Zhu, W. Y. Shih, W.H. Shih, Enhanced detection resonance frequency shift of a piezoelectric microcantilever sensor by a DC bias electric field in humidity detection, Sensors and Actuators B 138 (2009) 1-4.

[28] A.L. Beardslee, A.M. Addous, K.S. Demirci, O. Brand, S.M. Heinrich, F.Josse, Geometrical Optimization of Resonant Cantilevers Vibrating in In-Plane Flexural Modes, Sensors, IEEE (2010) $1996-1999$

[29] C. Vancura, I. Dufour, S. M. Heinrich, F. Josse, A. Hierlemann, Analysis of resonating microcantilevers operating in a viscous liquid environment, Sensors and Actuators A, 141 (2008) 4351.

[30] C. Vancura, J. Lichtenberg, A. Hierlemann, F. Josse, Characterization of magnetically actuated resonant cantilevers in viscous fluids, Appl. Phys. Lett. 87 (2005) 162510.

[31] D. R. Caudwell, J. P. M. Trusler, V. Vesovic, W. A. Wakeham, The Viscosity and Density of nDodecane and n-Octadecane at Pressures up to $200 \mathrm{MPa}$ and Temperatures up to $473 \mathrm{~K}$, International Journal of Thermophysics 25 September 2004 No. 5.

\section{Vitae}

Georges Dubourg was born in Bordeaux (France) in 1982. He received the engineer degree in electronics from the University of Sciences and Technologies in Bordeaux. Since 2009, he is working on organic microcantilevers for biochemical sensor applications at IMS laboratory, University of Sciences and technologies of Bordeaux as a PhD student.

Isabelle Dufour graduated from Ecole Normale Supérieure de Cachan in 1990 and received the Ph.D. and H.D.R. degrees in engineering science from the University of Paris-Sud, Orsay, France, in 1993 and 2000, respectively. She was a CNRS research fellow from 1994 to 2007, first in Cachan working on the modelling of electrostatic actuators (micromotors, micropumps) and then after 2000 in Bordeaux working on microcantilever-based chemical sensors. She is currently Professor of electrical engineering at the University of Bordeaux and her research interests are in the areas of microcantilever-based sensors for chemical detection, rheological measurements and materials characterisation.

From 1982 to 1993 Claude Pellet was a researcher at the "Institut d'Electronique Fondamentale" from the university Paris XI-Orsay, where he has studied the deposition of thin film by ion beam sputtering. He joined university of Bordeaux as full professor in 1993. His work focuses on micro-technology, micro-system development (humidity sensor), assembly technology, micro-systems reliability

Cédric Ayela was born in Tarbes (France) in 1981. He received the engineer degree in electronics from the national institute of applied sciences in Toulouse and a $\mathrm{PhD}$ degree in 2007 on piezoelectric silicon-based micromembranes for the label-free detection of biological molecules at the Laboratory for Analysis and Architecture of Systems (LAAS-CNRS) in Toulouse. He recently integrated (2009) the French national center for scientific research (CNRS) as a full researcher at IMS laboratory in Bordeaux (France). He is concentrating his researches on developing the emerging field of organic MEMS: fabrication, characterization, and application as bio/chemical sensors. 


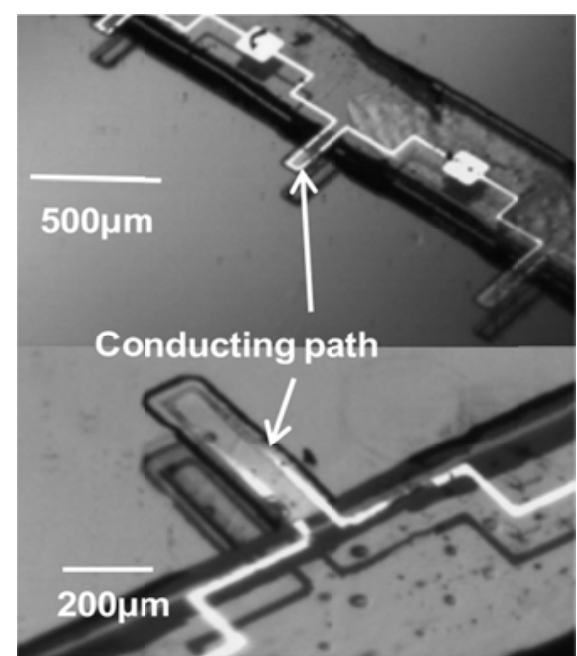

Fig. 1. Optical images of SU-8 microcantilever resonators.

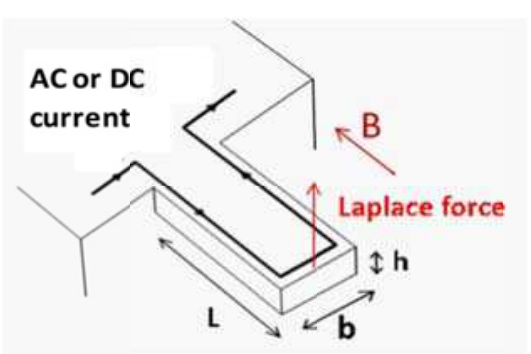

Fig. 2. Schematic of an electromagnetically actuated cantilever.

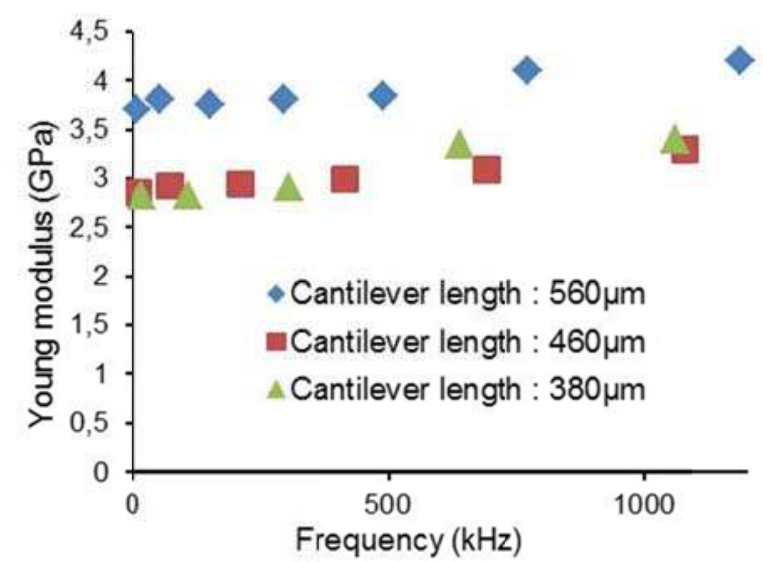

Fig. 3. Young's modulus for different flexural resonant modes of microcantilevers characterized by a width of $150 \mu \mathrm{m}$ and a thickness of $9 \mu \mathrm{m}$. 


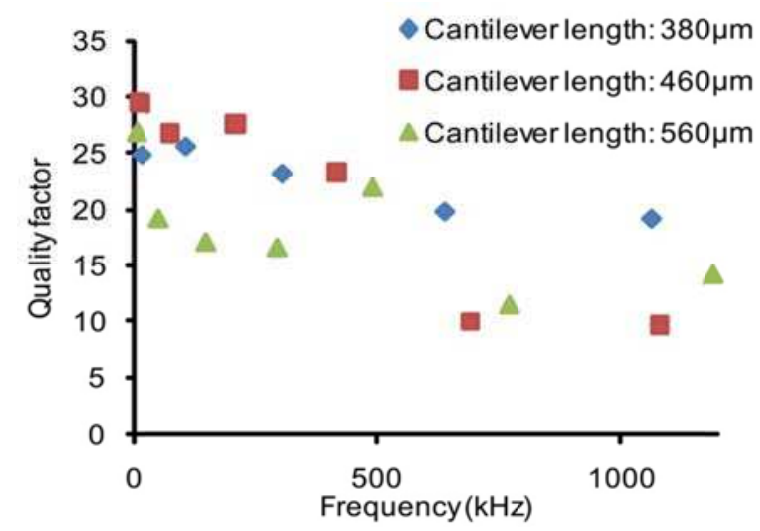

Fig. 4. Quality factor versus resonant frequency in air for different lengths of SU-8 cantilevers characterized by a common width of $150 \mu \mathrm{m}$ and a thickness of $9 \mu \mathrm{m}$.

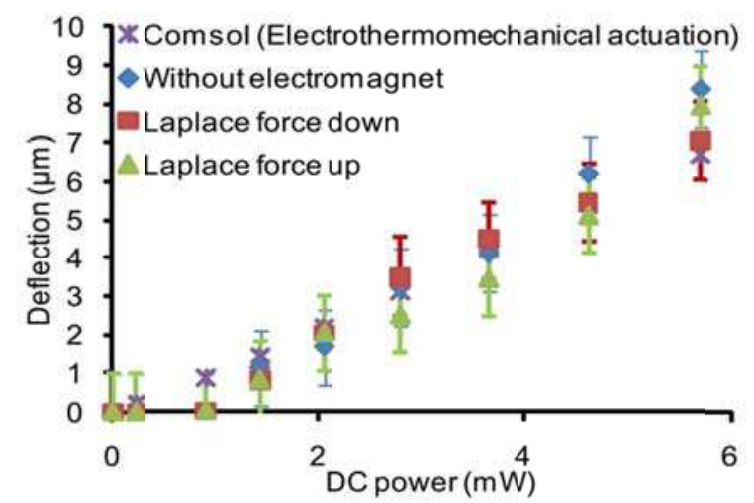

Fig. 5. Static deflection of a SU-8 cantilever characterized by a length of $380 \mu \mathrm{m}$, a width of $150 \mu \mathrm{m}$ and a thickness of $9 \mu \mathrm{m}$.

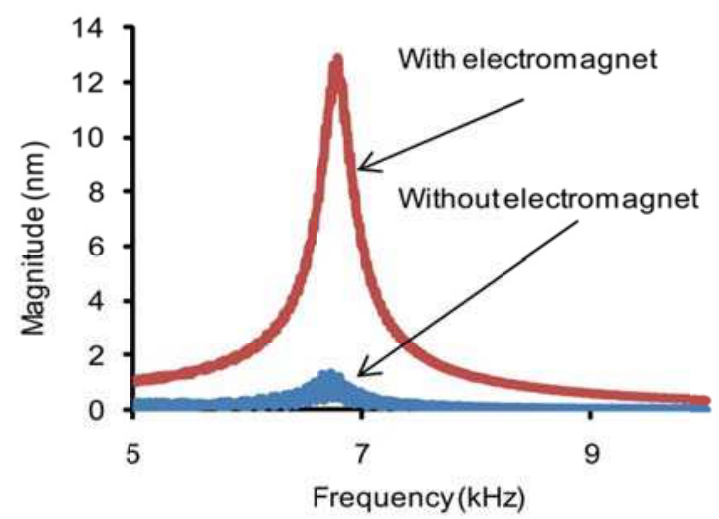

Fig. 6. Measurement of the $1^{\text {st }}$ flexural resonant mode with and without magnet for a structure characterized by a length of $600 \mu \mathrm{m}$, a width of $150 \mu \mathrm{m}$ and a thickness of $9 \mu \mathrm{m}$. Measurements have been realized with an $\mathrm{AC}$ current of $\mathrm{I}_{\max }=30 \mu \mathrm{A}$ and without $\mathrm{DC}$ current. 


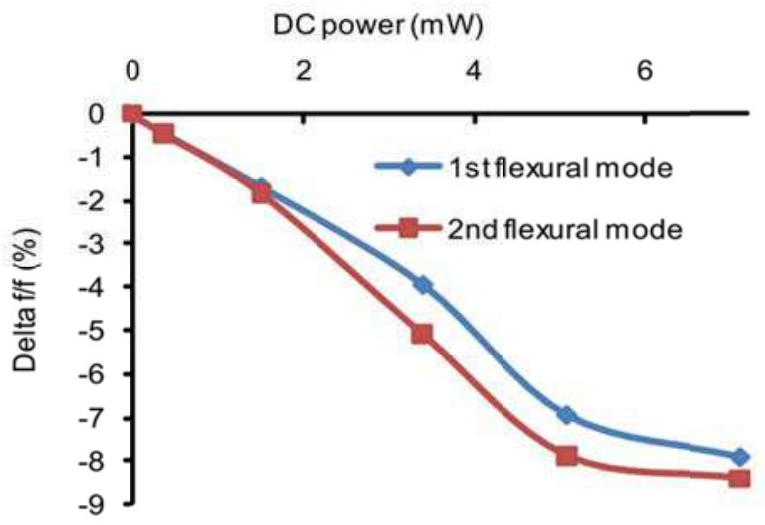

Fig. 7. Evolution of the resonant frequency as function of applied DC power for a structure characterized by a length of $380 \mu \mathrm{m}$, width of $150 \mu \mathrm{m}$ and a thickness of $9 \mu \mathrm{m}$.

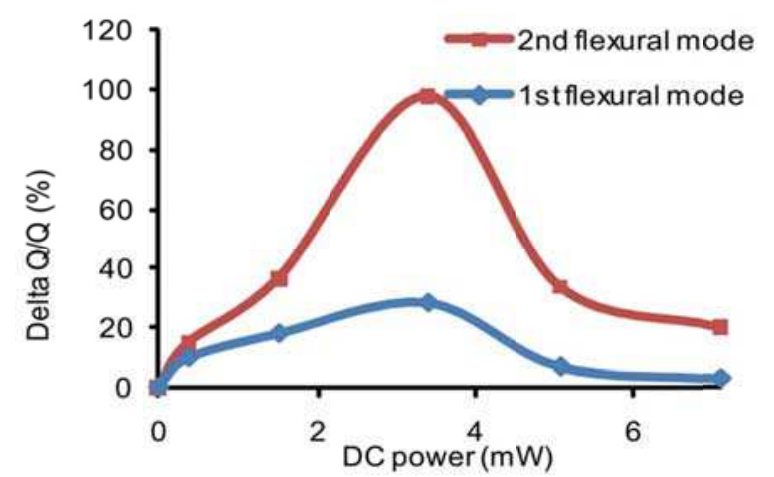

Fig. 8. Evolution of the quality factor with an increasing DC power applied to a microcantilever characterized by a length of $380 \mu \mathrm{m}$, a width of $150 \mu \mathrm{m}$ and a thickness of $9 \mu \mathrm{m}$. The initial quality factors of the resonant structures are 16 and 18 for the first flexural mode and the second flexural mode respectively.

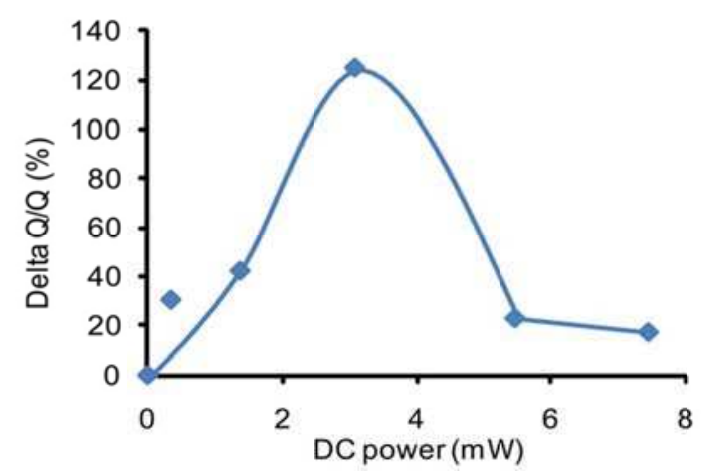

Fig. 9. Evolution of the quality factor with applied DC power in dodecane for the second flexural mode of a microcantilever characterized by a length of $380 \mu \mathrm{m}$, a width of $150 \mu \mathrm{m}$ and a thickness of $9 \mu \mathrm{m}$. The initial quality factor of the organic resonator is 4.8 . 